\title{
Equalization of optical nonlinear waveform distortion using SVM-based digital signal processing
}

\author{
Yuki Sato $^{1}$, Takeru Kyono ${ }^{1}$, Kai Ikuta ${ }^{1}$, Yuichiro Kurokawa ${ }^{1}$, \\ and Moriya Nakamura ${ }^{1, \text { a) }}$ \\ ${ }^{1}$ School of Science and Technology, Meiji University \\ 1-1-1 Higashimita, Tama-ku, Kawasaki, Kanagawa 214-8571, Japan \\ a)m_naka@meiji.ac.jp
}

Abstract: We proposed a novel nonlinear equalization scheme using a support vector machine (SVM) for optical fiber communication systems. The scheme works even when the received symbols are completely distorted on a constellation map. Whereas conventional SVM-based nonlinear equalization is performed on a two-dimensional (2D) in-phase-quadrature (IQ)-plane, our proposed scheme uses the SVM in a higher dimensional signal space after tapped delay lines. By employing the proposed scheme, we can enlarge the separation between the distorted symbols. We investigated the basic performance using simulated optical fiber transmission of 10-GSymbol/s 16QAM signals. The receiver sensitivity was improved by $2 \mathrm{~dB}$ at a BER of $10^{-5}$.

Keywords: optical communications, optical nonlinearity, nonlinear equalization, digital signal processing, SVM

Classification: Fiber-Optic Transmission for Communications

\section{References}

[1] K. Kikuchi, "Fundamentals of coherent optical fiber communications," $J$. Lightw. Technol, vol. 34, no. 1, pp. 157-179, Jan. 2016. DOI: 10.1109/jlt.2015. 2463719

[2] Y. Wang, S. Okamoto, K. Kasai, M. Yoshida, and M. Nakazawa, "Singlechannel $200 \mathrm{Gbit} / \mathrm{s}, 10 \mathrm{Gsymbol} / \mathrm{s}-1024$ QAM injection-locked coherent transmission over $160 \mathrm{~km}$ with a pilot-assisted adaptive equalizer," Opt. Express, vol. 26, no. 13, pp. 17015-17024, June 2018. DOI: 10.1364/oe.26.017015

[3] S. Owaki and M. Nakamura, "Equalization of optical nonlinear waveform distortion using neural-network based digital signal processing," Optoelectronics and Communications Conference (OECC2016), WA2-40, July 2016.

[4] S. Owaki, Y. Fukumoto, T. Sakamoto, N. Yamamoto, and M. Nakamura, "Experimental demonstration of SPM compensation based on digital signal processing using a three-layer neural-network for 40-Gbit/s optical 16QAM signal," IEICE Commun. Express, vol. 7, no. 1, pp. 13-18, Jan. 2018. DOI: 10.1587/comex.2017xb10148

[5] T. Kyono, Y. Otsuka, Y. Fukumoto, S. Owaki, and M. Nakamura, "Computational-complexity comparison of artificial neural network and 
Volterra series transfer function for optical nonlinearity compensation with time- and frequency-domain dispersion equalization," European Conference on Optical Communication (ECOC2018), Th2.28, Sept. 2018. DOI: 10.1109/ecoc. 2018.8535153

[6] J. Estarán, R. Rios-Müller, M.A. Mestre, F. Jorge, H. Mardoyan, A. Konczykowska, J.-Y. Dupuy, and S. Bigo, "Artificial neural networks for linear and non-linear impairment mitigation in high-baudrate IM/DD systems," European Conference on Optical Communication (ECOC2016), M.2.B.2, Sept. 2016.

[7] V. Kamalov, L. Jovanovski, V. Vusirikala, S. Zhang, F. Yaman, K. Nakamura, T. Inoue, E. Mateo, and Y. Inada, "Evolution from 8QAM live traffic to PS 64QAM with neural-network based nonlinearity compensation on $11000 \mathrm{~km}$ open subsea cable," Optical Fiber Communication Conference (OFC2018), Th4D.5, March 2018. DOI: 10.1364/ofc.2018.th4d.5

[8] M. Nakamura, M. Glick, A.K. Srivastava, and Y. Akasaka, "Optical nonlinearity compensation using artificial neural-network-based digital signal processing," Proc. SPIE 11308, Metro and Data Center Optical Networks and Short-Reach Links III, 113080N, Jan. 2020. DOI: 10.1117/12.2543325

[9] B. Schölkopf, K.-K Sung, C.J.C. Burges, F. Girosi, P. Niyogi, T. Poggio, and V. Vapnik, "Comparing support vector machines with Gaussian kernels to radial basis function classifiers," IEEE Trans. Signal Process., vol. 45, no. 11, pp. 2758-2765, Nov. 1997. DOI: 10.1109/78.650102

[10] T. Nguyen, S. Mhatli, E. Giacoumidis, L. V. Compernolle, M. Wulipart, and P. Mégret, "Fiber nonlinearity equalizer based on support vector classification for coherent optical OFDM," IEEE Photon. J., vol. 8, no. 2, 7802009, April 2016. DOI: 10.1109/jphot.2016.2528886

[11] Y. Cui, M. Zhang, D. Wang, S. Liu, Z. Li, and G.-K. Chang, "Bit-based support vector machine nonlinear detector for millimeter-wave radio-over-fiber mobile fronthaul systems," Opt. Express, vol. 25, no. 21, pp. 26186-26197, Oct. 2017. DOI: $10.1364 /$ oe.25.026186

[12] J. Zhang, W. Chen, M. Gao, and G. Shen, "Mitigating fiber nonlinearity using support vector machine with genetic algorithm," Optoelectronics and Communications Conference (OECC2017), P3-151, July 2017. DOI: 10.1109/cleopr. 2017.8118715

[13] C. Wang, G. Chen, H. Wang, L. Sun, J. Du, Z. He, K. Xu, and B. Liu, "SVM classification comparison for QAM modulated optical interconnection," Asia Communications and Photonics Conference (ACP2018), Su4C.5, Oct. 2018. DOI: 10.1109/acp.2018.8596155

[14] Y. Sato, T. Kyono, K. Ikuta, Y. Kurokawa, and M. Nakamura, "SVM-based nonlinearity equalization for optical communication systems," 2020 International Conference on Emerging Technologies for Communications (ICETC2020), I11, Dec. 2020.

\section{Introduction}

Digital coherent technology has increased the performance of optical fiber communication systems [1]. The technology has realized high receiver sensitivity and multilevel modulation formats such as quadrature amplitude modulation (QAM), which can realize high spectral efficiency [2]. However, the waveform of a QAM signal is distorted by nonlinear effects such as self-phase modulation (SPM) and cross phase modulation (XPM), because the signal power varies according to the trans- 
mitted symbols, resulting in a large peak-to-average power ratio (PAPR). Recently, artificial neural network (ANN)-based digital nonlinear equalizers have been receiving a lot of attention due to their nonlinear-compensation capabilities $[3,4,5,6,7]$. An ANN can approximately express arbitrary nonlinear continuous functions by a summation of many threshold functions [8]. On the other hand, a support vector machine (SVM) is a known method of achieving data classification with hyperplanes in the data space [9]. Some SVM-based nonlinear equalizers have already been investigated $[10,11,12,13]$. In these reported methods, however, the SVMclassifications were performed on the two-dimensional (2D) in-phase-quadrature (IQ)-plane of the transmitted symbols. Therefore, when inter-symbol overlap is caused by serious nonlinear distortion, there is insufficient inter-symbol separation for the SVM-classification. In our previous study, we proposed a novel SVM-based nonlinear equalization scheme [14]. The 2D space of the IQ-plane is mapped into a higher-dimensional signal space using tapped-delay lines. The hyperplanes in the higher-dimensional signal space classify the distorted symbols. In this paper, we investigated the basic performance of the proposed scheme using simulated optical fiber transmission of 10-GSymbol/s 16QAM signals. We evaluated the bit error rate (BER) performance and compared it with the conventional scheme.

\section{Proposed SVM-based nonlinear equalizer}

An SVM is a kind of machine learning algorithm used for pattern recognition. Data can be classified using hyperplanes after a learning process [9]. Figure 1(a) schematically shows a conventional SVM-based nonlinear equalizer. Linear equalization using a finite impulse response (FIR) filter is applied to compensate for the waveform distortion caused by chromatic dispersion of the optical fiber. This linear equalization, performed prior to the SVM-classification, is usually needed to enlarge the inter-symbol separation on the IQ-plane [10].

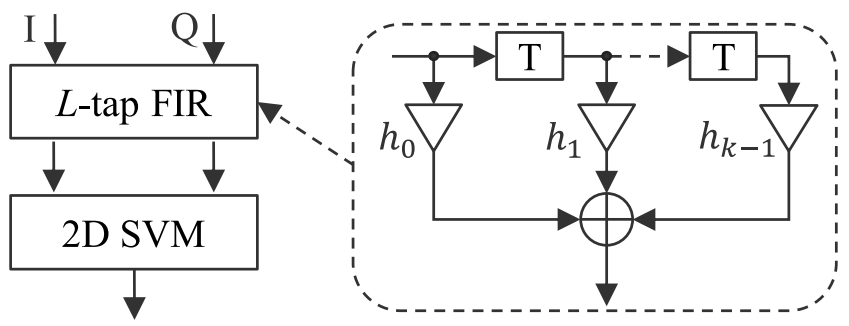

(a) Conventional SVM-based equalizer

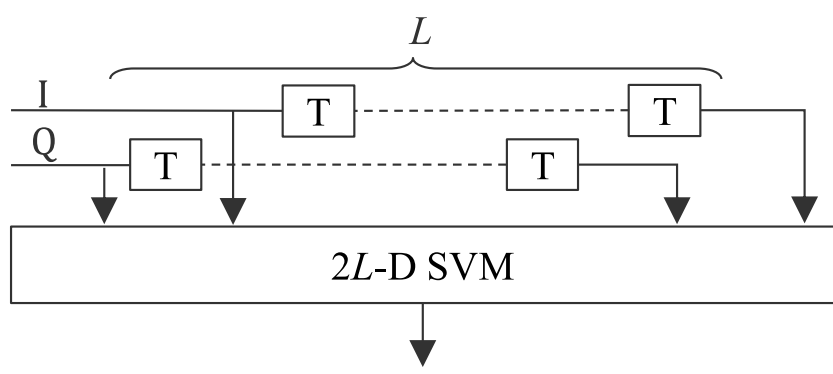

(b) Proposed SVM-based equalizer

() IEICE 2021

DOI: 10.1587/comex.2021ETL0022 Received February 10, 2021 Accepted March 10, 2021 Publicized April 9, 2021 
After the linear equalization, nonlinear classification by the $2 D S V M$ is performed on the IQ-plane without using tapped delay lines. This construction limits the nonlinear equalization performance because the linear equalization cannot give enough inter-symbol separation when the waveform is seriously distorted by nonlinear effects. Figure 1(b) shows the construction of our proposed SVM-based nonlinear equalizer. I- and Q-components of the distorted signal after the transmission are fed to a $2 L$-dimensional SVM ( $2 L-D S V M)$ through two tapped delay lines with a tap length of $L$. Therefore, linear and nonlinear equalizations are performed by the SVM-classification in the $2 L$-D signal space at the same time. In this case, the nonlinear classification can be performed using tapped delay lines.

\section{System setup}

Figure 2 shows the optical fiber transmission system used in the numerical simulation to investigate the nonlinear equalizer. 10-G symbol/s 16QAM signals were generated by modulating $2^{11}-1$ length PRBS and transmitted through $100-\mathrm{km}$ standard single mode fiber (SSMF). The wavelength of the laser diode (LD) was $1550 \mathrm{~nm}$. The loss parameter and dispersion coefficient of the SSMF were $0.2 \mathrm{~dB} / \mathrm{km}$ and 16.75 $\mathrm{ps} / \mathrm{nm} / \mathrm{km}$, respectively. The input power to the SSMF was $10 \mathrm{dBm}$, from erbiumdoped fiber amplifiers (EDFAs) with a noise figure of $5 \mathrm{~dB}$. After the transmission, the optical signal was received by optical homodyne detection using an optical $90^{\circ}$ hybrid and balanced photodetectors (BPDs). Here, we assumed that the local oscillator (LO) was ideally synchronized to the optical signal. The thermal power density of the BPDs was $1.0 \times 10^{-22} \mathrm{~W} / \mathrm{Hz}$. After optical homodyne detection, SVM-based equalization was performed in a digital signal processor (DSP). The number of taps was set to 7 . We employed the Gaussian radial basis function (RBF) as the kernel function [9]. The grid search method was used to optimize the kernel parameters. The equalization performance was evaluated by using the BER.

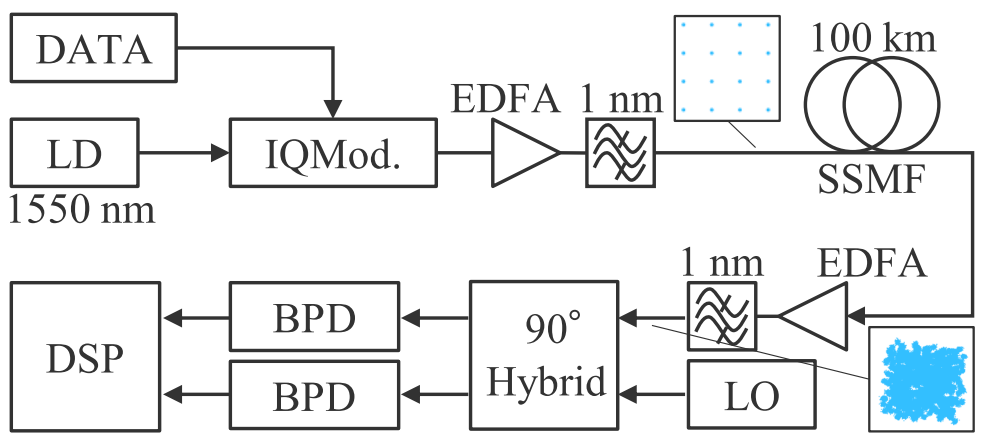

Fig. 2. System setup of 16QAM transmission.

\section{Results and discussion}

First, the conventional SVM-based equalization scheme was performed for comparison. Figures 3(a) and (b) show the constellations obtained with a back-to-back (BtB) setup and after $100 \mathrm{~km}$ transmission, respectively. As shown in Fig. 3(b), the transmitted signal was completely distorted by chromatic dispersion and SPM. 
The classification result obtained using the 2D SVM is also shown in the same figure. However, the SVM could not classify the transmitted symbols due to the serious signal distortion. The BER characteristics are denoted by black diamonds in Fig. 3(d). Figure 3(c) shows the constellation after the linear equalization using a 7-tap FIR filter. In this case, the 2D SVM could classify the symbols. However, sufficient inter-symbol separation was not obtained due to the residual nonlinear distortion. The BER characteristics are denoted by red circles in Fig. 3(d). The receiver sensitivity at a BER of $10^{-5}$ was about $-25 \mathrm{dBm}$. Next, we performed the proposed SVM-based equalization using tapped delay lines with a tap length of 7 and 14D SVM. The distorted signal on the 2D IQ-plane was mapped to the 14D signal space. The 14D SVM classified the distorted signal in the 14D space. The BER characteristics are denoted by blue crosses in Fig. 3(d). In this case, the receiver sensitivity at a BER of $10^{-5}$ was about $-27 \mathrm{dBm}$, which was a sensitivity improvement of $2 \mathrm{~dB}$.

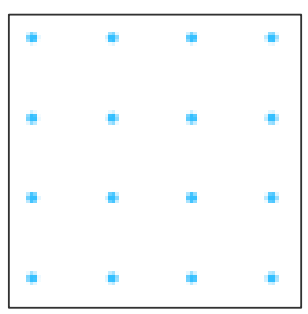

(a)

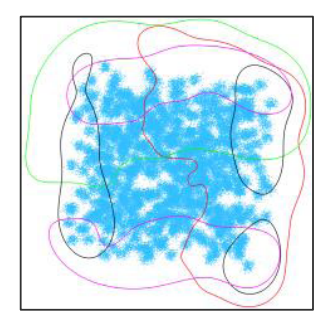

(b)

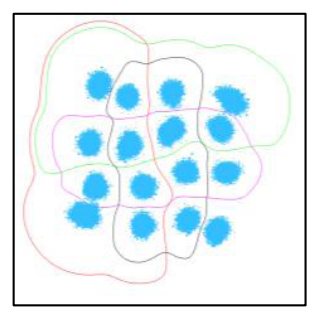

(c)

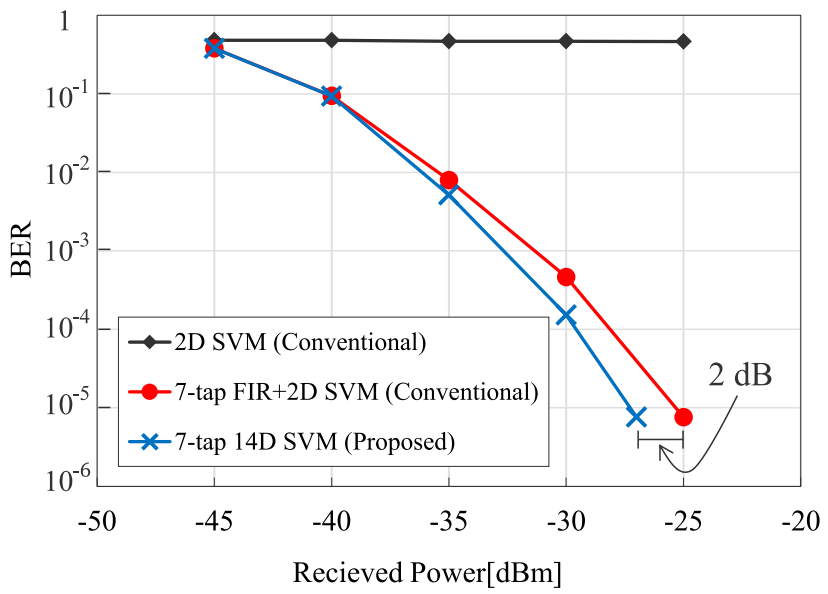

(d)

Fig. 3. (a) Constellation in BtB setup. (b) Constellation after transmission and classification by the 2D SVM. (c) Constellation after linear equalization and classification by the 2D SVM. (d) BER characteristics.

\section{Conclusion}

We proposed a novel SVM-based nonlinear equalization scheme that enables nonlinear classification using tapped delay lines. We investigated the performance of the proposed scheme using simulated optical fiber transmission of 10-GSymbol/s 
16QAM signals. We confirmed that the proposed scheme outperformed the conventional SVM-based equalizer.

\section{Acknowledgments}

This work was supported by JSPS KAKENHI Grant Number 20K05367 and the Fujikura Foundation. 